\title{
Analysis of Weight-Loss Outcomes Following Laparoscopic One Anastomosis Duodenal Switch
}

\author{
Amar Vennapusa1", Ramakanth Bhargav Panchangam², Charita Kesara', Gitika Raj Vyshnavi Vanta', \\ Aishwarya Reddy Sannareddy'
}

'Department of Obesity \& Diabetes Surgery, Dr. Amar Bariatric \& Metabolic Center, Hyderabad, India

${ }^{2}$ Department of Endocrine and Diabetes Surgery, Endocare Hospital, Vijayawada, India

${ }^{*}$ Corresponding author:

Dr. Amar Vennapusa FNB (MAS),

MS (Gen), DNB (Gen), MRCSEd

Chief Consultant Bariatric and

Metabolic Surgeon,

Dr. Amar Bariatric \& Metabolic Center

Plot No -1299D, First Floor, Green

View Plaza, Beside Indian Oil Fuel

Station, Opposite to KBR Park,

Road No $-1 \& 68$ Junction

Jubilee Hills, Hyderabad - 33, India

website: uww.drVamar.com

E-mail: drVamar@gmail.com
Received: 22.12 .2020

Accepted: 24.03.2021

\section{Rezumat}

Efectele laparoscopic one anastomosis duodenal switch asupra scăderii ponderale

Laparoscopic one anastomosis duodenal switch (OADS) este una dintre cele mai eficiente interventii chirurgicale bariatrice care vizează simplificarea procedurii de comutare duodenală şi reducerea malabsorbției fără a compromite eficacitatea scăderii ponderale. Acest studiu a analizat scăderea ponderală la pacienții la care s-a practicat interventia chirurgicală $\mathrm{OADS} \mathrm{cu}$ anastomoză gastro-ileală la $\geq 300 \mathrm{~cm}$ de cec.

Metode: Între iunie 2016 şi noiembrie 2020, 150 de pacienți care au fost supuşi unei intervenții chirurgicale OADS cu un segment intestinal comun $\geq 300 \mathrm{~cm}$ au fost incluşi în studiu. Scăderea ponderală la aceşti pacienți a fost analizată retrospectiv la 6 luni şi la 1 an postoperator şi a fost corelată cu diferite variabile perioperatorii, precum lungimile ansei biliopancreatice şi ale segmetelui intestinal comun.

Rezultate:La 6 luni şi la 1 an, \%TWL (total weight loss) a fost de $30 \%$ şi $39,4 \%$, iar $\% \mathrm{EWL}$ (excess weight loss) a fost de $75 \%$ şi, respectiv, 98,8\%. Scăderea poderală, reducerea IMC şi \%TWL au fost direct proportionale cu greutatea preoperatorie şi IMC, în timp ce $\% \mathrm{EWL}$ a fost invers proportională cu aceste variabile. IMC preoperator s-a dovedit a fi un predictor independent al reducerii IMC şi al \%EWL. Scăderea ponderală şi reducerea IMC $\mathrm{au}$ fost, de asemenea, direct proporționale cu lungimea ansei biliopancreatice. \%TWL a fost direct proporțională cu lungimea ansei biliopancreatice la 1 an postoperator. Vârsta, sexul şi 
prezența diabetului nu au avut influențat în mod semnificativ rata scăderii ponderale. Rata de succes (\%TWL $\geq 25 \%)$ a fost de $88,9 \%$ la 6 luni şi de $99,2 \%$ la 1 an postoperator.

Concluzii: Operatia OADS cu un segment intestinal comun $\geq 300 \mathrm{~cm}$ lungime pare a fi extrem de eficientă în ceea ce priveşte scăderea ponderală. Greutatea preoperatorie, IMC şi lungimea ansei biliopancreatice s-au dovedit a fi predictori semnificativi ai scăderii ponderale în urma acestei intervenții chirurgicale.

Cuvinte cheie: obezitate, diabet, scădere ponderală, chirurgie bariatrică, chirurgie metabolică

\section{Abstract}

Background: Laparoscopic one anastomosis duodenal switch (OADS) is one of the most effective bariatric surgeries aimed at simplifying duodenal switch procedure and reducing malabsorption without compromising weight-loss efficacy. This study analyzed weight-loss outcomes following OADS surgery with a common channel $\geq 300 \mathrm{~cm}$.

Methodology:Between June 2016 and November 2020, 150 patients who underwent OADS surgery with a common channel $\geq 300 \mathrm{~cm}$ were included in the study. The weight-loss outcomes of these patients at six months and one year after surgery were retrospectively studied with respect to various perioperative variables including biliopancreatic limb (BPL) and common channel lengths. Results: At six months and one year after surgery, \%TWL was $30 \%, 39.4 \%$, and \%EWL was $75 \%$, $98.8 \%$, respectively. The BMI loss, weight loss and \%TWL were directly proportional to preoperative $\mathrm{BMI}$ and weight, while the \%EWL was inversely proportional to these variables. Preoperative BMI was found to be an independent predictor of BMI loss and \%EWL. The BMI loss, and weight loss were also directly proportional to BPL length. The \%TWL was directly proportional to BPL length at one year after surgery. Age, gender and diabetes did not have significant effects on the weight-loss outcomes. The success rate ( $\% \mathrm{TWL} \geq 25 \%)$ was $88.9 \%$ and $99.2 \%$ at six months and one year after surgery, respectively.

Conclusions: OADS surgery using a common channel $\geq 300 \mathrm{~cm}$ appears to be highly effective for weight loss. Preoperative BMI, weight and BPL length were found to be significant predictors of weight-loss outcomes following this surgery.

Key words: obesity, diabetes, weight loss, bariatric surgery, metabolic surgery

\section{Introduction}

Obesity, along with its associated comorbidities, is a major global health problem that increases mortality and reduces lifespan. Dietary modifications and exercise have been shown to be ineffective in managing obesity in the long term, especially when obesity is severe. In contrast, bariatric surgery offers sustainable weight loss with minimal complications. The effects of bariatric surgery are due to physiological and hormonal alterations in the body, rather than to food restriction and malabsorption (1).

Duodenal switch (DS) surgery is the most effective surgery to treat obesity. However, it accounts for less than $1 \%$ of the total bariatric surgeries performed throughout the world (2). While technical complexity is one reason for this low rate of performance, severe malabsorption is another important reason the surgery is uncommon. Laparoscopic loop modifications of the DS use a 200 to $250 \mathrm{~cm}$ common channel. However, even though loop 
modifications simplify DS, malabsorption remains a concern (3). Neichoy et al. (4), used a $300 \mathrm{~cm}$ common channel, as opposed to the $100 \mathrm{~cm}$ common channel length in DS. By increasing this length to $300 \mathrm{~cm}$, the risk of short bowel syndrome is (theoretically) reduced, and the length provides a margin of error in cases of inaccurate bowel measurement (5).

The available literature regarding one anastomosis duodenal switch (OADS) surgery is limited. Weight-loss predictors are commonly evaluated after routine bariatric surgeries such as laparoscopic sleeve gastrectomy (SG) and laparoscopic Roux-en-Y gastric bypass (RYGB). However, there is limited literature regarding the various factors that can predict weight loss following OADS surgery. In addition, the effect of biliopancreatic limb (BPL) and common channel lengths on the weight-loss outcomes and hypoalbuminemia following OADS surgery were not well studied. Therefore, in this study we analyzed our experiences with OADS surgery using a common channel $\geq 300 \mathrm{~cm}$ (Fig. 1) to ascertain its efficacy in terms of weight loss and various perioperative factors that may predict weightloss outcomes. In addition, we analyzed the effect of limb lengths on hypoalbuminemia.

\section{Methodology}

Indian patients with severe obesity (Body mass index $-\mathrm{BMI} \geq 30 \mathrm{~kg} / \mathrm{m}^{2}$ ) who underwent OADS surgeries between June 2016 and November 2020 were retrospectively studied. Main inclusion criteria was OADS surgeries with common channel $\geq 300 \mathrm{~cm}$. Those who underwent OADS as a revision surgery were excluded. In our center, bariatric and metabolic surgeries were offered primarily to the patients with $\mathrm{BMI} \geq 35 \mathrm{~kg} / \mathrm{m}^{2}\left(\mathrm{BMI} \geq 32.5 \mathrm{~kg} / \mathrm{m}^{2}\right.$ in Indians) with type 2 diabetes or other comorbid conditions and patients with $\mathrm{BMI} \geq 40 \mathrm{~kg} / \mathrm{m}^{2}$ $\left(\mathrm{BMI} \geq 37.5 \mathrm{~kg} / \mathrm{m}^{2}\right.$ in Indians) even without any comorbid conditions. However, these surgeries were also offered as an alternative option to the patients with $\mathrm{BMI} \geq 30 \mathrm{~kg} / \mathrm{m}^{2}$ and $<35 \mathrm{~kg} / \mathrm{m}^{2}\left(\mathrm{BMI} \geq 27.5 \mathrm{~kg} / \mathrm{m}^{2}\right.$ and $<32.5 \mathrm{~kg} / \mathrm{m}^{2}$

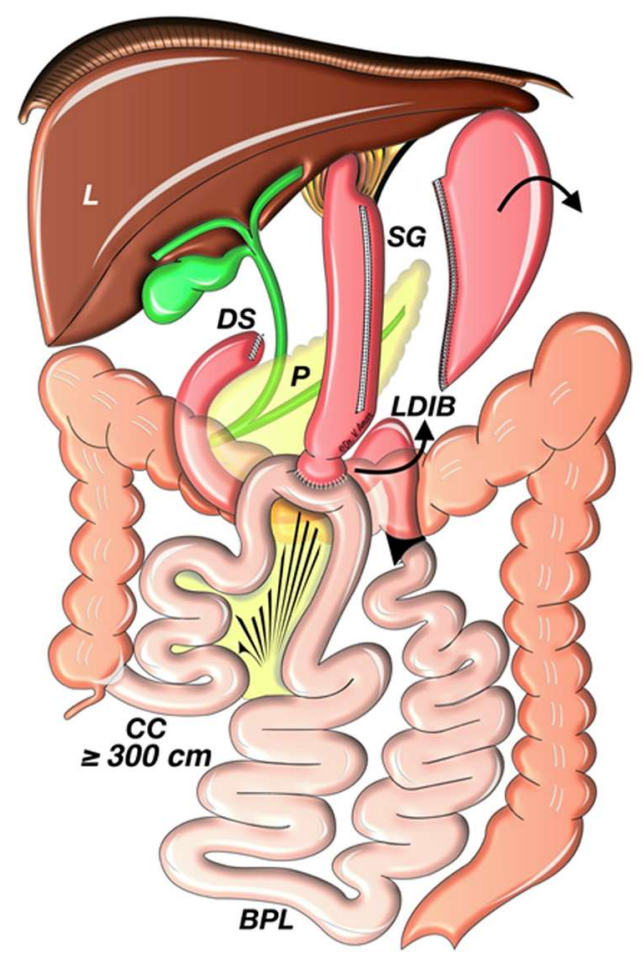

Figure 1. Diagram of one anastomosis duodenal switch Liver - L, Gastric Sleeve - SG, Pancreas - P, Stump of the duodenum - DS, Loop duodenoileal anastomosis (bypass) LDIB, Biliopancreatic (Afferent) limb - BPL, Common channel (Efferent limb) - CC.

Figure 1 is drawn by the corresponding author.

in Indians) who failed to achieve substantial and durable weight loss with nonsurgical treatments. Institutional review board approval was obtained prior to the start of the study. All the participants in the study provided informed written consent. Relevant clinical and follow-up data was documented in a master data sheet.

After constructing a lax gastric sleeve using a 38 Fr calibration tube, the first part of the duodenum was transected and anastomosed to the ileum in an ante-colic, end-to-side loop fashion. In OADS surgery with fixed common channel - OADS (FCC) surgery, common channel was $350 / 300 \mathrm{~cm}$ regardless of the total length of the small intestine. In OADS surgery with fixed ratio bypass - OADS (FRB) surgery, a fixed percentage of the small intestine was bypassed. During the initial phase of the study period (from June 2016 to September 2019), mainly OADS (FCC) surgery was performed 
using a 350/300 cm common channel. The selection of $350 / 300 \mathrm{~cm}$ was arbitrarily based on the total small intestinal length. A $350 \mathrm{~cm}$ FCC was preferentially used when the total length of the small intestine was $\geq 700 \mathrm{~cm}$. When the total small intestinal length was < $700 \mathrm{~cm}$, a $300 \mathrm{~cm}$ FCC was preferred. When we observed an increased risk of protein energy malnutrition with BPL of $>55 \%$ of the total small intestinal length, we started performing OADS (FRB) surgery by restricting the BPL to $\leq 55 \%$. Selection of the $\%$ of BPL $(45 \%, 50 \%$ or $55 \%)$ was based on several preoperative variables. Specifically, we used higher \%BPL in patients with lower ages, higher BMIs, the presence of comorbidities, or longer total small intestinal lengths.

BMI loss, weight loss, \%TWL, and \%EWL (with $25 \mathrm{~kg} / \mathrm{m}^{2} \mathrm{BMI}$ as a reference point) were calculated at six months and one year after surgery. These parameters were studied in the age, gender, and BMI groups and in the groups based on the diabetes status (diabetes vs. prediabetes vs. no diabetes). Success rate was defined as a \%TWL $\geq 25 \%$ at the different follow-up periods(6). When based on the $\%$ EWL, success was arbitrarily defined as a $\% \mathrm{EWL} \geq 60 \%(7)$.

Complete diabetes remission was defined as $\mathrm{HbA} 1 \mathrm{C}<6 \%$ with no medications required for glycemic control (8). Partial diabetes remission was defined as $\mathrm{HbA} 1 \mathrm{C} \geq 6$ and $<6.5 \%$ with no medications needed for glycemic control. Diabetes improvement was defined as a statistically significant reduction in $\mathrm{HbA} 1 \mathrm{C}$ that did not meet the criteria for remission, or a decrease in the requirements for antidiabetic medications (8). Serum albumin and total protein levels were studied before surgery, six months and one year after surgery. Serum albumin $<3.5 \mathrm{~g} / \mathrm{dL}$ was considered as hypoalbuminemia (9). Based on serum levels (g/dL), hypoalbuminemia was categorised as mild (3 - 3.49), moderate (2.5 -2.99) and severe $(<2.5)(10)$.

Paired sample t-tests were undertaken to find differences in weight parameters at six months and one year after surgery. Independent sample t-tests and one-way ANOVAs were performed to analyze differences in the weight-loss parameters between different groups. Simple linear regression and multiple regression analyses were performed to find the effects of patient's age, gender, BPL length, common channel length, and preoperative BMI, weight and diabetes status on weight parameters at six months and one year after surgery. Effects of these variables on postoperative success rates were studied using binary regression. Significance was defined as a p-value < 0.05. IBM SPSS Version 26 (IBM Corp., Armonk, NY, USA) was used for all statistical analyses.

\section{Results}

A total of 152 patients underwent OADS surgery with a common channel $\geq 300 \mathrm{~cm}$ as a primary surgery for obesity between June 2016 and November 2020. Of these patients, 150 met the inclusion criteria and were analyzed for this study. The mean preoperative age of the patients was $39.9 \pm 10.3$ years, with 30 patients $(20 \%) \geq 50$ years of age. The male/female ratio was $82: 68$. Twenty-six $(17.3 \%)$ patients had a BMI $\geq 50 \mathrm{~kg} / \mathrm{m}^{2}$. Seventy-three $(48.7 \%)$ patients had type 2 diabetes and $46(30.7 \%)$ had prediabetes. FCC of 350 and $300 \mathrm{~cm}$ were used in 102 and 16 patients, respectively. OADS (FRB) surgery was performed in 32 patients (the \%BPL was $45 \%$ in $1,50 \%$ in $18,55 \%$ in 10 , and $60 \%$ in 3 patients). The average common channel length was $349.9 \pm 26.3(300-450) \mathrm{cm}$. Since November 2017, the length of the total small intestine was measured in 120 patients. The total small intestine was $790.8 \pm 105.6$ $(610-1165) \mathrm{cm}$ and the average BPL was 441 $\pm 93.9(270-815) \mathrm{cm}$ in these 120 patients.

The mean BMI and weight of patients at the different follow-up periods are shown in Table 1 and Fig. 2. Significant changes in BMI and weight were observed at six months in comparison to before surgery and at one year after surgery in comparison to before surgery and six months after surgery. BMI loss, weight loss, \%TWL, and \%EWL at six months and one year after surgery are shown in Table 1 , 
Table 1. Weight loss parameters

\begin{tabular}{|c|c|c|c|c|c|}
\hline Variable & Number & Preoperative & Six months & One year & PST $^{\mathrm{a}}$ \\
\hline Weight (kg) & 150 & $122.9 \pm 24.2$ & - & - & - \\
\hline Weight (kg) & 135 & $122.8 \pm 24.8$ & $85.8 \pm 16.8$ & - & $<0.001$ \\
\hline Weight (kg) & 119 & $121.4 \pm 24.8$ & $85 \pm 16.7$ & $72.8 \pm 12.5$ & $<0.001$ \\
\hline $\mathrm{BMI}\left(\mathrm{kg} / \mathrm{m}^{2}\right)$ & 150 & $44.4 \pm 7.4$ & - & - & - \\
\hline BMI $\left(\mathrm{kg} / \mathrm{m}^{2}\right)$ & 135 & $44.2 \pm 7.6$ & $30.9 \pm 5.1$ & - & $<0.001$ \\
\hline BMI $\left(\mathrm{kg} / \mathrm{m}^{2}\right)$ & 119 & $44 \pm 7.7$ & $30.8 \pm 5.2$ & $26.4 \pm 3.9$ & $<0.001$ \\
\hline Weight loss (kg) & 135 & - & $37 \pm 10.3$ & - & - \\
\hline Weight loss (kg) & 119 & - & $36.5 \pm 10.3$ & $48.6 \pm 15.8$ & $<0.001$ \\
\hline BMI loss $\left(\mathrm{kg} / \mathrm{m}^{2}\right)$ & 135 & - & $13.3 \pm 3.4$ & - & - \\
\hline BMI loss $\left(\mathrm{kg} / \mathrm{m}^{2}\right)$ & 119 & - & $13.2 \pm 3.4$ & $17.6 \pm 5.2$ & $<0.001$ \\
\hline \%TWL (\%) & 135 & - & $30 \pm 4.3$ & - & - \\
\hline$\%$ TWL (\%) & 119 & - & $29.8 \pm 4.3$ & $39.4 \pm 6.1$ & $<0.001$ \\
\hline \%EWL (\%) & 135 & - & $75 \pm 20.6$ & - & - \\
\hline$\%$ EWL (\%) & 119 & - & $75.7 \pm 21.2$ & $98.8 \pm 22.6$ & $<0.001$ \\
\hline
\end{tabular}

Significance ( $p$-value) in paired samples t-test

Fig 2. All of these parameters were significantly more at one year after surgery in comparison to six months after surgery. Weight-loss parameters in various groups at six months and one year after surgery are shown in Tables 2 and 3.

There were no significant differences in weight-loss parameters in the groups based on age. While males lost significantly more weight than females at six months and one year after surgery, other weight-loss parameters were not significantly different between these groups (Table 2). A one-way ANOVA test showed no difference in weight-loss outcomes in patient groups based on preoperative diabetes status (diabetes vs. prediabetes vs. no diabetes) (Table 3).

The one-way ANOVA analyses showed that there was significantly more BMI loss, weight loss in patients with a preoperative $\mathrm{BMI} \geq 50$ $\mathrm{kg} / \mathrm{m}^{2}$ compared to those with a BMI of 40 to 49.9 and $<40$, and in patients with a BMI between 40 to 49.9 compared to those with a $\mathrm{BMI}<40$ at six months and one year after surgery. Patients with a preoperative $\mathrm{BMI} \geq 50$ $\mathrm{kg} / \mathrm{m}^{2}$ had significantly higher \%TWL compared to those with a BMI between 40 to 49.9 and $<40$ at one year after surgery; however, this difference was not significant at six months after surgery. \%TWL was similar in BMI groups of 40 to 49.9 and ' $<40$ ' at six months and one year after surgery. Patients with BMI < 40 had higher \%EWL compared to those with BMI of 40 to 49.9 and $\geq 50$ at six months and one year after surgery. Similarly, patients with BMI of 40 to 49.9 had higher \%EWL compared to those with BMI of $\geq 50$ at six months and one year after surgery (Table 3).

Table 2. Variance in weight loss response in age and gender groups

\begin{tabular}{|c|c|c|c|c|c|c|c|c|c|c|}
\hline \multirow[t]{2}{*}{ Follow-up } & \multirow[t]{2}{*}{ Groups } & \multirow[t]{2}{*}{$\mathbf{N}$} & \multicolumn{2}{|c|}{ Weight loss (kg) } & \multicolumn{2}{|c|}{ BMI loss $\left(\mathrm{kg} / \mathrm{m}^{2}\right)$} & \multicolumn{2}{|c|}{ \%TWL (\%) } & \multicolumn{2}{|c|}{ \%EWL (\%) } \\
\hline & & & Mean \pm SD & IST $^{a}$ & Mean \pm SD & IST $^{a}$ & Mean \pm SD & IST $^{a}$ & Mean \pm SD & IST $^{\mathbf{a}}$ \\
\hline Six months & $\begin{array}{l}\text { Age }(<50 \text { years }) \\
\text { Age }(\geq 50 \text { years })\end{array}$ & $\begin{array}{c}108 \\
27\end{array}$ & $\begin{array}{l}37.1 \pm 10.2 \\
36.6 \pm 11\end{array}$ & 0.819 & $\begin{array}{l}13.2 \pm 3.2 \\
13.8 \pm 4\end{array}$ & 0.436 & $\begin{array}{l}29.9 \pm 4.4 \\
30.3 \pm 3.8\end{array}$ & 0.663 & $\begin{array}{l}75.4 \pm 21.4 \\
73.4 \pm 17.1\end{array}$ & 0.664 \\
\hline One year & $\begin{array}{l}\text { Age ( }<50 \text { years) } \\
\text { Age ( } \geq 50 \text { years })\end{array}$ & $\begin{array}{l}96 \\
23 \\
\end{array}$ & $\begin{array}{l}48.7 \pm 15.2 \\
48.3 \pm 18.5\end{array}$ & 0.918 & $\begin{array}{l}17.4 \pm 4.8 \\
18.2 \pm 6.9 \\
\end{array}$ & 0.523 & $\begin{array}{l}39.4 \pm 5.8 \\
39.5 \pm 7.3\end{array}$ & 0.959 & $\begin{array}{l}99.6 \pm 22.8 \\
95.9 \pm 21.8\end{array}$ & 0.483 \\
\hline Six months & $\begin{array}{l}\text { Males } \\
\text { Females }\end{array}$ & $\begin{array}{l}74 \\
61\end{array}$ & $\begin{array}{l}40.4 \pm 10.1 \\
32.9 \pm 9.1\end{array}$ & $<0.001$ & $\begin{array}{l}13.6 \pm 3.3 \\
12.9 \pm 3.4\end{array}$ & 0.245 & $\begin{array}{l}30.4 \pm 4.4 \\
29.4 \pm 4.2\end{array}$ & 0.147 & $\begin{array}{l}75.1 \pm 19.6 \\
74.9 \pm 21.8\end{array}$ & 0.955 \\
\hline One year & $\begin{array}{l}\text { Males } \\
\text { Females }\end{array}$ & $\begin{array}{l}63 \\
56\end{array}$ & $\begin{array}{l}52.9 \pm 16 \\
43.9 \pm 14.2\end{array}$ & 0.002 & $\begin{array}{l}17.9 \pm 5.2 \\
17.2 \pm 5.3\end{array}$ & 0.451 & $\begin{array}{l}40.1 \pm 6.3 \\
38.7 \pm 5.8\end{array}$ & 0.210 & $\begin{array}{l}99.3 \pm 20.2 \\
98.3 \pm 25.2\end{array}$ & 0.822 \\
\hline
\end{tabular}

aSignificance (p-value) in independent samples t-test; SD - standard deviation 
Figure 2. Weight loss variables at different time intervals
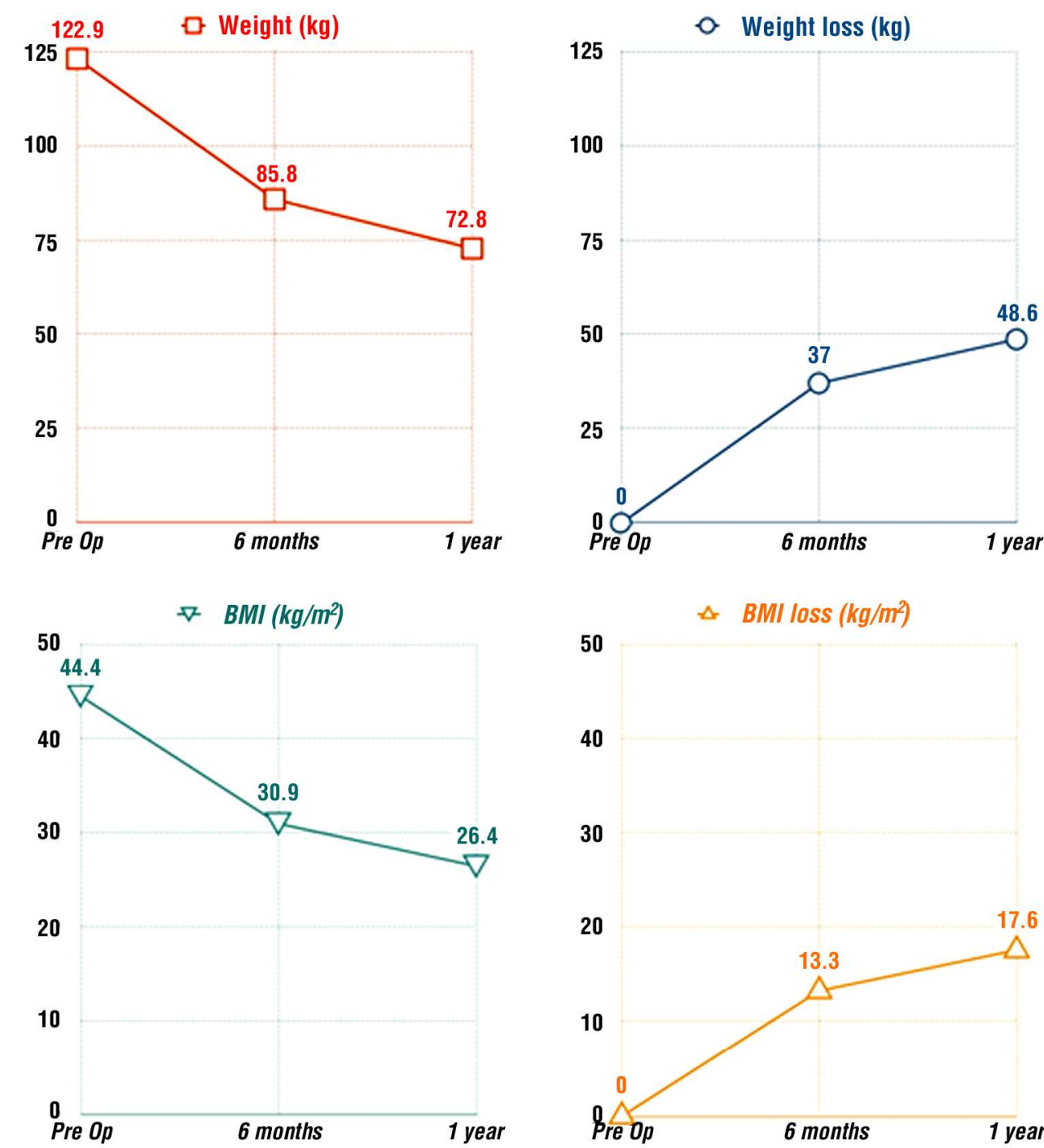

50

$\triangle \quad$ BMI loss $\left(\mathrm{kg} / \mathrm{m}^{2}\right)$

40

30

20

17.6

10
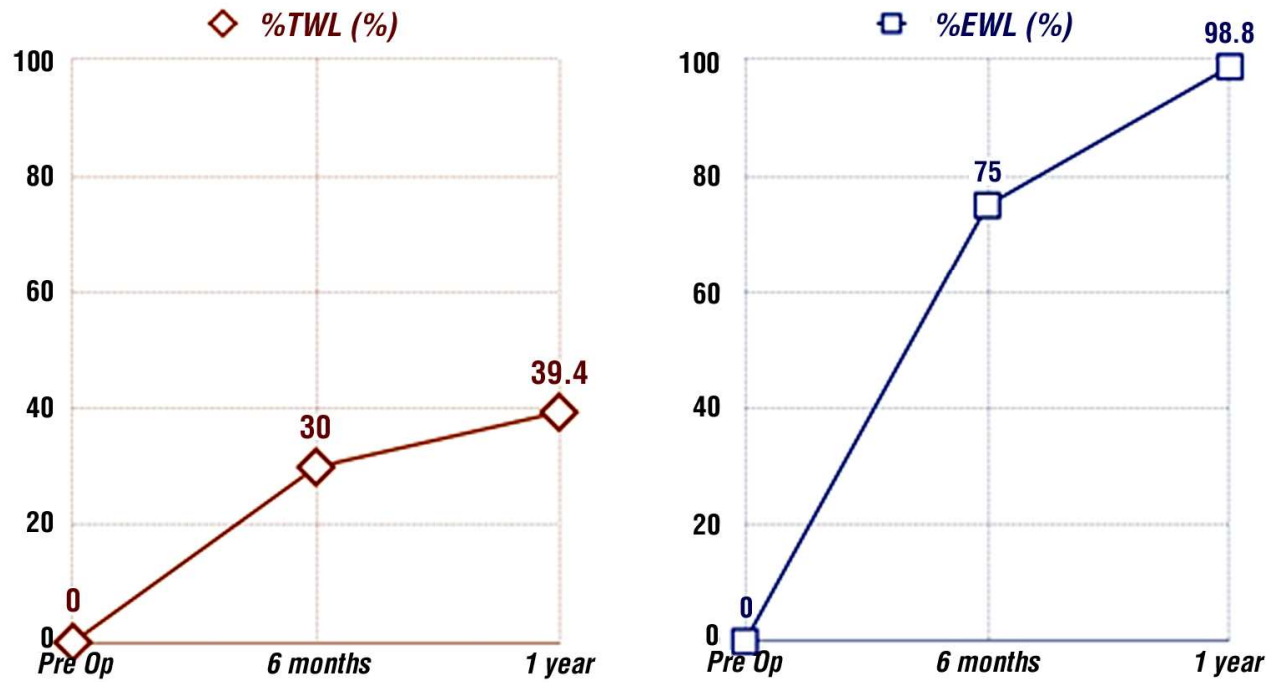
Table 3. Variance in weight loss response in BMl and diabetes groups

\begin{tabular}{|c|c|c|c|c|c|c|c|c|c|c|}
\hline \multirow[t]{2}{*}{ Follow-up } & \multirow[t]{2}{*}{ Groups } & \multirow[t]{2}{*}{$\mathrm{N}$} & \multicolumn{2}{|c|}{ Weight loss (kg) } & \multicolumn{2}{|c|}{ BMI loss (kg/m²) } & \multicolumn{2}{|c|}{ \%TWL (\%) } & \multicolumn{2}{|c|}{ \%EWL (\%) } \\
\hline & & & Mean \pm SD & Sig. & Mean \pm SD & Sig. & Mean \pm SD & Sig. & Mean \pm SD & Sig. \\
\hline \multirow{3}{*}{$\begin{array}{l}\mathrm{BMl}\left(\mathrm{kg} / \mathrm{m}^{2}\right) \\
\text { at six } \\
\text { months }\end{array}$} & $\mathrm{BMI}<40 \mathrm{~kg} / \mathrm{m}^{2}$ & 42 & $30.3 \pm 7$ & $<0.001^{a}$ & $11.1 \pm 2.1$ & $<0.001^{a}$ & $30.2 \pm 4.9$ & $0.607^{a}$ & $98.3 \pm 19.6$ & $<0.001^{\mathrm{a}}$ \\
\hline & BMI $40-49.9 \mathrm{~kg} / \mathrm{m}^{2}$ & 70 & $37.1 \pm 7.1$ & $<0.001^{\mathrm{b}}$ & $13.2 \pm 2.1$ & $<0.001^{\mathrm{b}}$ & $29.4 \pm 4$ & $0.165^{b}$ & $67 \pm 8.7$ & $0.003^{b}$ \\
\hline & $\mathrm{BMI} \geq 50 \mathrm{~kg} / \mathrm{m}^{2}$ & 23 & $48.9 \pm 12.8$ & $<0.001^{c}$ & $17.8 \pm 4$ & $<0.001^{c}$ & $31.3 \pm 3.9$ & $0.595^{c}$ & $56.6 \pm 6$ & $<0.001^{\mathrm{c}}$ \\
\hline \multirow{3}{*}{$\begin{array}{l}\mathrm{BMI}\left(\mathrm{kg} / \mathrm{m}^{2}\right) \\
\text { at one year }\end{array}$} & $\mathrm{BMl}<40 \mathrm{~kg} / \mathrm{m}^{2}$ & 40 & $37.4 \pm 8.4$ & $<0.001^{a}$ & $13.7 \pm 2.6$ & $<0.001^{a}$ & $37.5 \pm 5.7$ & $0.259^{a}$ & $122.3 \pm 19.9$ & $<0.001^{\mathrm{a}}$ \\
\hline & BMl $40-49.9 \mathrm{~kg} / \mathrm{m}^{2}$ & 58 & $49.6 \pm 9.8$ & $<0.001^{\mathrm{b}}$ & $17.7 \pm 2.9$ & $<0.001^{\mathrm{b}}$ & $39.4 \pm 5.1$ & $0.019^{b}$ & $89.8 \pm 10.9$ & $0.014^{b}$ \\
\hline & $\mathrm{BMI} \geq 50 \mathrm{~kg} / \mathrm{m}^{2}$ & 21 & $67.3 \pm 21$ & $<0.001^{\mathrm{c}}$ & $24.6 \pm 6.5$ & $<0.001^{\mathrm{c}}$ & $43.4 \pm 7.5$ & $0.001^{c}$ & $79.1 \pm 12.5$ & $<0.001^{\circ}$ \\
\hline \multirow{3}{*}{$\begin{array}{l}\text { Diabetes } \\
\text { status at } \\
\text { six months }\end{array}$} & Diabetes & 69 & $36.5 \pm 11$ & $0.613^{\mathrm{d}}$ & $13.2 \pm 3.8$ & $0.645^{d}$ & $29.4 \pm 4.1$ & $0.057^{d}$ & $73.3 \pm 19.5$ & $0.412^{\mathrm{d}}$ \\
\hline & Prediabetes & 38 & $38.4 \pm 9.9$ & $0.651^{e}$ & $13.8 \pm 2.9$ & $0.287^{e}$ & $31.4 \pm 4.6$ & $0.15^{\mathrm{e}}$ & $79.3 \pm 24.7$ & $0.467^{e}$ \\
\hline & No diabetes & 28 & $36.4 \pm 9$ & $0.999^{f}$ & $12.8 \pm 2.6$ & $0.783^{f}$ & $29.4 \pm 4.1$ & $0.999^{\prime}$ & $73.3 \pm 16.4$ & $1^{\dagger}$ \\
\hline \multirow{3}{*}{$\begin{array}{l}\text { Diabetes } \\
\text { status at } \\
\text { one year }\end{array}$} & Diabetes & 65 & $48.1 \pm 17.2$ & $0.859^{d}$ & $17.5 \pm 6$ & $0.882^{d}$ & $38.4 \pm 6.6$ & $0.076^{d}$ & $95.3 \pm 22.1$ & $0.285^{d}$ \\
\hline & Prediabetes & 35 & $49.7 \pm 13.2$ & $0.968^{\circ}$ & $18 \pm 3.8$ & $0.727^{e}$ & $41 \pm 4.8$ & $0.826^{e}$ & $103.2 \pm 25.8$ & $1^{\mathrm{e}}$ \\
\hline & No diabetes & 19 & $48.7 \pm 16$ & $0.99^{\dagger}$ & $17 \pm 4.6$ & $0.93^{f}$ & $40 \pm 6.2$ & $0.607^{1}$ & $103.1 \pm 16$ & $0.218^{f}$ \\
\hline
\end{tabular}

One way ANOVA post hoc tests - Significance (p-value) in ${ }^{\mathrm{a}, \mathrm{b}, \mathrm{c}, \mathrm{T}}$ Tukey HSD test and ${ }^{\mathrm{d}, \mathrm{e}, \mathrm{f}} \mathrm{Games}$-Howell test. SD - Standard Deviation;

${ }^{\mathrm{a} B M I}<40$ vs. $40-49.9 \mathrm{~kg} / \mathrm{m}^{2},{ }^{\mathrm{b}} \mathrm{BMl} 40-49.9$ vs. $\geq 50 \mathrm{~kg} / \mathrm{m}^{2},{ }^{\mathrm{c}} \mathrm{BMl} \geq 50 \mathrm{vs}$. $<40 \mathrm{~kg} / \mathrm{m}^{2}$

DDiabetes vs. prediabetes, ${ }^{\mathrm{e} P}$ rediabetes vs. no diabetes, ${ }^{\mathrm{N}}$ No diabetes vs. diabetes

A simple linear regression analysis showed that preoperative BMI and weight significantly predicted the BMI loss, weight loss, \%TWL, and \%EWL at six months and one year after surgery, while patient's age did not predict these parameters (Table 4 ). Both preoperative BMI and weight were directly proportional to the BMI loss, weight loss, and \%TWL and inversely proportional to \%EWL at six months and one year after surgery. Multiple regression analysis showed that the preoperative BMI was an independent predictor of BMI loss and $\% \mathrm{EWL}$, and that the preoperative weight was an independent predictor of weight loss at six months and one year after surgery. However, neither preoperative BMI, nor preoperative weight were found to be independent predictors of \%TWL at six months and one year after surgery. BPL length was directly proportional to BMI loss and weight loss at six months and one year after surgery, and to \%TWL at one year after surgery, but no significant association between BPL length and \%EWL was found. Multiple regression analysis showed that BPL length independently predicted BMI loss and weight loss at one year after surgery. However, it did not independently predict \%TWL (Table 4 ). While a simple linear regression analysis showed that the common channel length was directly proportional to weight

Table 4. Significance of simple linear and multiple regression analysis

\begin{tabular}{|c|c|c|c|c|c|c|c|c|c|}
\hline \multirow[t]{2}{*}{ Follow-up } & \multirow[t]{2}{*}{ Independent Variable } & \multicolumn{2}{|c|}{ Weight loss } & \multicolumn{2}{|c|}{ BMI loss } & \multicolumn{2}{|c|}{$\%$ TWL } & \multicolumn{2}{|c|}{$\% E W L$} \\
\hline & & SLR $^{a}$ & $M_{R} A^{b}$ & SLR $^{a}$ & $M_{R A}$ & SLR $^{\mathrm{a}}$ & $M_{R A}$ & SLR $^{\mathrm{a}}$ & MRA $^{\mathrm{b}}$ \\
\hline \multirow[t]{7}{*}{ six months } & Age & 0.131 & 0.796 & 0.873 & 0.880 & 0.700 & 0.966 & 0.604 & 0.460 \\
\hline & Gender $^{\mathrm{c}}$ & - & 0.311 & - & 0.305 & - & 0.279 & - & 0.254 \\
\hline & Preoperative Weight & $<0.001$ & $<0.001$ & $<0.001$ & 0.815 & 0.017 & 0.760 & $<0.001$ & 0.274 \\
\hline & Preoperative BMI & $<0.001$ & 0.283 & $<0.001$ & $<0.001$ & 0.012 & 0.343 & $<0.001$ & 0.003 \\
\hline & Diabetes $^{d}$ & - & 0.251 & - & 0.314 & - & 0.263 & - & 0.563 \\
\hline & $\mathrm{BPL}^{\mathrm{e}}$ & $<0.001$ & 0.094 & 0.009 & 0.099 & 0.081 & 0.143 & 0.688 & 0.070 \\
\hline & $\mathrm{CC}^{\mathrm{f}}$ & 0.039 & 0.215 & 0.557 & 0.331 & 0.956 & 0.360 & 0.177 & 0.177 \\
\hline \multirow[t]{7}{*}{ one year } & Age & 0.219 & 0.909 & 0.814 & 0.910 & 0.265 & 0.714 & 0.184 & 0.363 \\
\hline & Gender $^{c}$ & - & 0.869 & - & 0.920 & - & 0.946 & - & 0.835 \\
\hline & Preoperative Weight & $<0.001$ & $<0.001$ & $<0.001$ & 0.254 & $<0.001$ & 0.289 & $<0.001$ & 0.836 \\
\hline & Preoperative BMI & $<0.001$ & 0.212 & $<0.001$ & $<0.001$ & $<0.001$ & 0.259 & $<0.001$ & 0.001 \\
\hline & Diabetes $^{d}$ & - & 0.015 & - & 0.020 & - & 0.020 & - & 0.288 \\
\hline & $\mathrm{BPL}^{\mathrm{e}}$ & $<0.001$ & 0.029 & 0.008 & 0.032 & 0.020 & 0.080 & 0.750 & 0.074 \\
\hline & $\mathrm{CC}^{f}$ & 0.062 & 0.233 & 0.460 & 0.290 & 0.329 & 0.422 & 0.601 & 0.300 \\
\hline
\end{tabular}

Significance ( $p$-value) in simple linear regression, ${ }^{\text {}}$ Significance ( $p$-value) in multiple regression analysis, ${ }^{c}$ Males vs. females, ${ }^{d}$ Diabetes vs. prediabetes vs. no diabetes, ${ }^{e}$ Biliopancreatic limb length, 'Common channel length 
change at six months after surgery, its significance was lost when BPL length was taken as its covariate in the multiple regression analysis and the significance of the BPL length was maintained.

The success rate (defined as $\% \mathrm{TWL} \geq 25 \%$ ) following OADS surgery was $88.9 \%$ and $99.2 \%$ at six months and one year after surgery, respectively. Univariate logistic regression showed that age was directly proportional to the success rate $(\% \mathrm{TWL} \geq 25 \%)$ at six months after surgery. When the success rate was defined as $\% \mathrm{EWL} \geq 60 \%$, these values were $76.3 \%$ and $98.3 \%$, respectively. Univariate logistic regression showed that preoperative BMI and weight were inversely proportional to the success rate $(\% \mathrm{EWL} \geq 60 \%)$ at six months after surgery. But no perioperative variable independently predicted success rate after surgery (Table 5). We did not use \%EWL $\geq 50 \%$ to define success rate as it was $100 \%$ at one year after surgery; thus, a binary logistic regression analysis could not be performed.

At six months and one year after surgery, diabetes remission was complete in $82.6 \%$ and $90.8 \%$, partial in $10.1 \%$ and $7.7 \%$ and diabetes improved in $7.3 \%$ and $1.5 \%$, respectively. None of the patients experienced diabetes deterioration (Table 6).

Before surgery, six months and one year after surgery, 119, 99 and 87 patients, respectively had their serum albumin and total protein values measured. Serum albumin levels $(\mathrm{g} / \mathrm{dL})$ were $4 \pm 0.4,3.7 \pm 0.4$ and $3.7 \pm 0.6$, while total protein levels $(\mathrm{g} / \mathrm{dL})$ were $7.3 \pm 0.5$, $6.7 \pm 0.6$, and $6.6 \pm 0.6$ before surgery, six months and one year after surgery, respectively. Decrease in these values was significant at six months and one year after surgery compared to before surgery. $7.6 \%, 22.2 \%$ and $31 \%$ of patients respectively had low albumin levels before surgery, six month and one year after surgery. Despite low albumin levels, 96\% and 93.1\% patients at six months and one year after surgery respectively, maintained levels $\geq$ $3 \mathrm{~g} / \mathrm{dL}$. Albumin levels $<2.5 \mathrm{~g} / \mathrm{dL}$ were found in $2 \%$ and $3.45 \%$ at six months and one year after surgery, respectively (Table 6).

The BPL length significantly predicted hypoalbuminemia at six months $(p=0.013)$ and one year $(p=0.004)$ after surgery. BPL length and hypoalbuminemia were directly proportional. Specifically, there was an increased rate of hypoalbuminemia when the BPL length was $>55 \%$ vs. $\leq 55 \%(28.1 \%$ vs. $14.3 \%$ at six months and $40.7 \%$ vs. $15.2 \%$ at one year after surgery, respectively), which was significant at one year after surgery. All the patients with moderate and severe hypoalbuminemia at six months and one year after surgery had a BPL length $>55 \%$. Patients with severe hypoalbuminemia experienced clinical manifestations such as pedal edema and ascites. Severe hypoalbuminemia occurred

Table 5. Significance with logistic regression analysis

\begin{tabular}{|c|c|c|c|c|c|}
\hline \multirow[t]{2}{*}{ Follow-up } & \multirow[t]{2}{*}{ Independent variable } & \multicolumn{2}{|c|}{$\%$ TWL $\geq 25$ vs. $<25$} & \multicolumn{2}{|c|}{$\% E W L \geq 60$ vs. $<60$} \\
\hline & & ULR $^{a}$ & MLR $^{b}$ & ULR $^{\mathrm{a}}$ & $\mathrm{MLR}^{\mathrm{b}}$ \\
\hline \multirow[t]{7}{*}{ six months } & Age & 0.024 & 0.128 & 0.073 & 0.255 \\
\hline & Gender $^{c}$ & 0.503 & 0.436 & 0.531 & 0.314 \\
\hline & Preoperative Weight & 0.648 & 0.738 & $<0.001$ & 0.286 \\
\hline & Preoperative BMI & 0.300 & 0.487 & $<0.001$ & 0.212 \\
\hline & Diabetes $^{d}$ & 0.835 & 0.876 & 0.433 & 0.901 \\
\hline & $\mathrm{BPL}^{\mathrm{e}}$ & 0.841 & 0.958 & 0.653 & 0.204 \\
\hline & $\mathrm{CC}^{\dagger}$ & 0.058 & 0.182 & 0.193 & 0.214 \\
\hline \multirow[t]{7}{*}{ one year } & Age & 0.576 & 1.000 & 0.117 & 0.454 \\
\hline & Gender $^{c}$ & 0.997 & 0.999 & 0.933 & 0.552 \\
\hline & Preoperative Weight & 0.313 & 1.000 & 0.624 & 0.767 \\
\hline & Preoperative BMI & 0.203 & 0.999 & 0.203 & 0.921 \\
\hline & Diabetes $^{d}$ & 1.000 & 1.000 & 1.000 & 1.000 \\
\hline & $\mathrm{BPL}^{\mathrm{e}}$ & 0.888 & 1.000 & 0.206 & 0.215 \\
\hline & $\mathrm{CC}^{\mathrm{f}}$ & 0.988 & 0.998 & 0.219 & 0.650 \\
\hline
\end{tabular}

a Significance ( $p$ value) in univariate logistic regression; ${ }^{\circ}$ Significance ( $p$ value) in multivariate logistic regression

'Males vs. females, 'Diabetes vs. prediabetes vs. no diabetes; 'Biliopancreatic limb length, 'Common channel length 
Table 6. Diabetes response and hypoalbuminemia

\begin{tabular}{lccccc}
\hline Diabetes response & Number & $\begin{array}{c}\text { Complete } \\
\text { remission }\end{array}$ & $\begin{array}{c}\text { Partial } \\
\text { remission }\end{array}$ & $\begin{array}{c}\text { Diabetes } \\
\text { improvement }\end{array}$ & $\begin{array}{c}\text { Diabetes } \\
\text { deterioration }\end{array}$ \\
\hline six months & 69 & $57(82.6 \%)$ & $7(10.1 \%)$ & $5(7.3 \%)$ & $0(0 \%)$ \\
\hline one year & 65 & $59(90.8 \%)$ & $5(7.7 \%)$ & $1(1.5 \%)$ & $0(0 \%)$ \\
\hline Albumin groups & Number & $\begin{array}{c}\text { Eualbuminemia } \\
(\geq \mathbf{3 . 5} \mathbf{g} / \mathbf{d L})\end{array}$ & $\begin{array}{c}\text { Mild } \\
\text { hypoalbuminemia } \\
(\mathbf{3}-\mathbf{3 . 4 9} \mathbf{g} / \mathbf{d L})\end{array}$ & $\begin{array}{c}\text { Moderate } \\
\text { hypoalbuminemia } \\
(\mathbf{2 . 5}-\mathbf{2 . 9 9} \mathbf{g} / \mathbf{d L})\end{array}$ & $\begin{array}{c}\text { Sevpere } \\
\text { hypoalbumineima } \\
(<\mathbf{2 . 5} \mathbf{g} / \mathbf{d L})\end{array}$ \\
\hline Preoperative & 119 & $110(92.4 \%)$ & $9(7.6 \%)$ & $0(0 \%)$ & $0(0 \%)$ \\
\hline six months & 99 & $77(77.8 \%)$ & $18(18.2 \%)$ & $2(2 \%)$ & $2(2 \%)$ \\
\hline one year & 87 & $60(69 \%)$ & $21(24.1 \%)$ & $3(3.45 \%)$ & $3(3.45 \%)$ \\
\hline
\end{tabular}

Values are mentioned as number (\%)

in three patients at one year after surgery and was treated using parenteral supplements, diet modifications and muscle strengthening exercises. Albumin levels improved in two patients while the third developed liver cirrhosis and lost life 13 months following surgery.

\section{Discussion}

Bariatric surgery has been shown to be superior to lifestyle modifications alone for achieving weight loss (11). SG surgery is the most commonly performed surgery (12), but weight gain following this surgery is significant in the long-term (13). RYGB surgery also has limitations such as the inability to monitor the remnant stomach endoscopically, an increased risk of calcium and iron deficiencies due to complete duodenal bypass, dumping due to the bypass of the pylorus, a lack of endoscopic access to the biliary tract, a marginal ulcer risk caused by the unopposed exposure of the jejunum to gastric juice, and internal hernias due to defects in the mesentery (14).

DS is very powerful in inducing durable weight loss (15). Since pylorus and initial 2 to $5 \mathrm{~cm}$ of the duodenum are not bypassed in DS, calcium and iron deficiencies are addressed to some extent, but an extensive intestinal bypass that leaves only $100 \mathrm{~cm}$ for absorption increases protein malabsorption, nutritional deficiencies, and renal stones (16). Thus, its popularity has decreased and is performed less than $1 \%$ of the time. Several loop modifications of DS have been introduced using various lengths of the common channel to address malabsorption associated with DS (17). These surgeries are collectively called SADI-S/OADS (single anastomosis duodenoileal bypass with sleeve/OADS). OADS surgery with a common channel length $\geq 300$ $\mathrm{cm}$ is aimed at reducing malabsorption without compromising weight-loss efficacy.

In our study, patients achieved significant BMI loss, weight loss, \%TWL and \%EWL at six months and one year after surgery, indicating that OADS surgery is a highly effective procedure for achieving significant weight loss. A study by Andres Sanchez et al. (18) showed a mean \%EWL of $94.7 \%$ following SADI-S surgery with a $200 \mathrm{~cm}$ common channel. In comparison, \%EWL was higher in our study $(p=0.048)$. Other studies have shown similar weight-loss responses after SADI-S and DS surgeries. For example, Moon et al. (19) demonstrated that \%TWL was similar after single anastomosis duodenal switch (SADS) and double anastomosis DS (DADS) surgeries. In that study, the authors found that \%TWL was $22.0 \%$ and $38.5 \%$ in the SADS surgery group and $20.2 \%$ and $38.0 \%$ in the DADS surgery group at six months and one year after surgery, respectively. Cottam et al. (20) compared stomach intestinal pylorus sparing surgery (SIPS) surgery with DS surgery and found no difference in the \%TWL and \%EWL in either procedure at six months and one year after surgery. In their study, \%EWL was $58.3 \%$ and $78.7 \%$ after SIPS surgery and $63.7 \%$ and $82.7 \%$ after DS surgery at six months and one year after surgery, respectively. Similarly, $\%$ TWL was $27.3 \%$ and $36 \%$ after SIPS surgery 
and $29.5 \%$ and $38.4 \%$ after DS surgery at six months and one year after surgery, respectively. Compared to their results following SIPS surgery, the \%TWL and \%EWL were higher in our study $(p<0.001)$. Various studies have reported a \%TWL ranging from $37 \%$ to $38.9 \%$ and a $\%$ EWL ranging from $61.6 \%$ to $95 \%$ at one year after various SADI-S/OADS surgeries (17). This variability may be related to differences in preoperative patient characteristics and BPL lengths in the different studies.

Various studies have shown that weightloss response is significantly better after SADI-S/OADS surgeries compared to conventional surgeries such as RYGB and SG. Enochs et al. (21) showed significantly more weight loss at 12 and 24 months following OADS surgery compared to RYGB and SG surgeries. According to Cottam et al., (22) loop DS surgery yielded better \%TWL and \%EWL compared to SG surgery at 3, 6, 9, 12, 15, and 18 months after surgery.

Factors predicting weight loss after SG and RYGB surgeries have been extensively investigated; however, there is limited available literature that has investigated factors predicting weight loss after loop DS surgeries such as SADI-S, OADS, SIPS, and SADS. Our results indicate that preoperative BMI and weight were directly proportional to BMI loss, weight loss, \%TWL, and inversely proportional to \%EWL. This suggests that patients with higher preoperative BMI and weight achieved significantly higher BMI loss, weight loss, \%TWL, while patients with lower preoperative BMI achieved significantly higher \%EWL. Our results also indicate that preoperative BMI was an independent predictor of BMI loss and \%EWL, but not of \%TWL, while preoperative weight was an independent predictor of weight change at six months and one year after surgery. Furthermore, a number of studies have shown that preoperative BMI is the most consistent predictor of postoperative weight-loss outcomes following bariatric surgery, with higher BMIs resulting in lower $\%$ EWLs (23-25).

Our analysis has also demonstrated that there was no difference in \%TWL and \%EWL after OADS surgery in gender, age and diabetes groups at six months and one year after surgery. This indicates that OADS surgery was equally effective in males and females, regardless of patient's age. While different investigations have demonstrated that weight-loss outcomes are inversely proportional to patient's age after standard surgeries such as RYGB, SG and one anastomosis gastric bypass (OAGB) $(23,25-29)$, our results indicate that age did not predict weight-loss outcomes after OADS surgery. This is an important finding, which emphasizes that while standard surgeries do not provide effective weight loss in patients $\geq 50$ years, OADS surgery may yield better results. Furthermore, the presence of diabetes did not affect the weight-loss response after OADS surgery. This is similar to Contreas et al. (26) who found that preoperative diabetes status did not affect weight loss after bariatric surgery.

BPL length was shown to predict BMI loss and weight loss at six months and one year after surgery and \%TWL at one year after surgery in our study, and patients with longer BPL lengths achieved greater weight loss. Roslin et al. (30) has suggested that higher weight-loss response is likely related to longer BPL lengths following SADS surgery and the direct emptying of undigested food into the distal ileum. The significant association shown between the common channel length and \%TWL at six months after surgery in our study was, in fact, the effect of BPL length and not the common channel length.

Our results found that preoperative BMI, weight and BPL length were significant factors that predicted weight-loss outcomes after OADS surgery. None of the other variables predicted weight-loss outcomes, possibly because OADS surgery was extremely effective as indicated by high BMI loss, weight loss, \%TWL and \%EWL.

Hormonal changes such as reduced ghrelin, increased glucagon-like peptide 1 (GLP1), and physiological changes including diversion of biliopancreatic juices and alterations in bile acid circulation and gut microbiota are 
responsible for weight loss after bariatric surgery (31). The roles of food restriction and malabsorption in weight loss is secondary. Alimentary anastomosis is placed in the distal ileum during OADS surgery and likely correlates with a potent ileal brake mediated by an enhanced secretion of GLP1 and peptide YY, which stimulates early satiety (32). Similarly, the longer the BPL length, the greater the weight loss (30). Therefore, the effects of OADS surgery were probably related to the direct emptying of nutrients into the ileum and long BPL lengths.

The weight-loss success rate following OADS surgery was $>98 \%$ at one year after surgery, and its high success rate dominated the influence of the predicting factors on this outcome. However, more patients and longer follow-up periods are needed to assess how different predictors affect the success rate after this surgery. Diabetes remission (complete and partial) was $>92 \%$ at six months and $>98 \%$ at one year after surgery. These findings indicate that OADS surgery was a highly effective metabolic surgery for diabetes remission. Severe hypoalbuminemia was $<3.5 \%$ at one year after surgery in our study. Enochs et al. (21) has reported albumin deficiency in 3.1\% of SADS surgery patients at one year after surgery.

The presence of pylorus and initial 2 to 5 $\mathrm{cm}$ of the duodenum in the food pathway, probably decrease malabsorption in OADS surgery. The pylorus controls gastric emptying, which allows bypassing longer length of small intestine with reduced risk of malabsorption (30). Bypassing a significant length of the small intestine was likely to have been the main reason for the marked hypoalbuminemia we observed, with reduced protein intake as a contributing factor. As the BPL length of > $55 \%$ significantly increased hypoalbuminemia in our patients, it is preferable to measure the total small intestinal length in all patients undergoing OADS surgery and restrict BPL to $\leq 55 \%$ to reduce the incidence of severe hypoalbuminemia. Our research was primarily intended to study weight-loss variables and hypoalbuminemia following OADS surgery. A detailed study of diabetes remission and vitamin and mineral deficiencies following this surgery was beyond the scope of this study.

\section{Conclusions}

Our OADS surgery technique entailed a loop modification of DS with a common channel $\geq$ $300 \mathrm{~cm}$. Our results showed that this surgery resulted in highly effective weight loss in patients suffering from obesity. Due to the extremely high BMI loss, weight loss, \%TWL, $\% \mathrm{EWL}$, and the success rate, age, gender, or diabetes could not predict weight-loss response after this surgery. However, preoperative BMI, weight, and BPL length significantly predicted weight-loss outcomes. Randomized controlled trials and multicenter studies with long-term follow-ups are needed to further analyze weight-loss response after this surgery.

\section{Conflicts of Interest}

We hereby declare that there are no hidden conflicts of interests either financial or plagiarism related or any other related to the clinical content and work of this manuscript.

\section{Ethics of Approval}

Our study complied with the ethical principles for medical research involving human subjects according to the Helsinki declaration.

\section{References}

1. Tam CS, Berthoud HR, Bueter M, Chakravarthy MV, Geliebter A, Hajnal A, et al. Could the mechanisms of bariatric surgery hold the key for novel therapies? report from a Pennington Scientific Symposium. Obes Rev. 2011; 12(11):984-94.

2. Angrisani L, Santonicola A, lovino P, Vitiello A, Higa K, Himpens J, et al. IFSO Worldwide Survey 2016: Primary, Endoluminal, and Revisional Procedures. Obes Surg. 2018;28(12):3783-3794.

3. Vilallonga R, Balibrea JM, Curell A, Gonzalez O, Caubet E, Ciudin A, et al. Technical Options for Malabsorption Issues After Single Anastomosis Duodenoileal Bypass with Sleeve Gastrectomy. Obes Surg. 2017;27(12): 3344-3348.

4. Neichoy BT, Schniederjan B, Cottam DR, Surve AK, Zaveri HM, Cottam A, et al. Stomach Intestinal Pylorus-Sparing Surgery for Morbid Obesity. JSLS. 2018 Jan-Mar;22(1). pii: e2017.00063.

5. Mitzman B, Cottam D, Goriparthi R, Cottam S, Zaveri H, Surve A, et al. 
Stomach Intestinal Pylorus Sparing (SIPS) Surgery for Morbid Obesity: Retrospective Analyses of Our Preliminary Experience. Obes Surg. 2016; 26:2098-2104

6. van de Laar AW, van Rijswijk AS, Kakar H, Bruin SC. Sensitivity and Specificity of 50\% Excess Weight Loss (50\%EWL) and Twelve Other Bariatric Criteria for Weight Loss Success. Obesity Surgery. 2018;28: 2297-2304.

7. Ortega E, Morinigo R, Flores L, Moize V, Rios M, Lacy AM, et al. Predictive factors of excess body weight loss 1-year after laparoscopic bariatric surgery. Surg Endosc. 2012;26:1744-1750.

8. Brethauer SA, Kim J, el Chaar M, Papasavas P, Eisenberg D, Rogers A, et al. Standardized outcomes reporting in metabolic and bariatric surgery. Surg Obes Relat Dis. 2015;11(3):489-506

9. Akirov A, Masri-Iraqi H, Atamna A, Shimon I. Low Albumin Levels Are Associated with Mortality Risk in Hospitalized Patients. Am J Med. 2017; 130(12):1465.e11-1465.e19.

10. Kuin C, den Ouden F, Brandts H, Deden L, Hazebroek E, van Borren M et al. Treatment of Severe Protein Malnutrition After Bariatric Surgery. Obes Surg. 2019;29:3095-3102.

11. Simonson DC, Halperin F, Foster K, Vernon A, Goldfine AB. Clinical and Patient-Centered Outcomes in Obese Patients With Type 2 Diabetes 3 Years After Randomization to Roux-en-Y Gastric Bypass Surgery Versus Intensive Lifestyle Management: The SLIMM-T2D Study. Diabetes Care. 2018;41(4): 670-679.

12. Angrisani L, Santonicola A, lovino P, Vitiello A, Zundel N, Buchwald H, et al. Bariatric Surgery and Endoluminal Procedures: IFSO Worldwide Survey 2014. Obes Surg. 2017;27(9):2279-2289.

13. Lauti M, Kularatna M, Hill AG, MacCormick AD. Weight Regain Following Sleeve Gastrectomy - a Systematic Review. Obes Surg. 2016;26:1326

14. Magouliotis DE, Tasiopoulou VS, Tzovaras G. One Anastomosis Gastric Bypass Versus Roux-en-Y Gastric Bypass for Morbid Obesity: an Updated Meta-Analysis. Obes Surg. 2019;29(9):2721-2730.

15. Copaescu C. Laparoscopic Biliopancreatic Diversion with Duodenal Switch - The Most Effective Operation for Type 2 Diabetes Mellitus. How I do it? Chirurgia (Bucur). 2018;113(5):704-711.

16. Homan J, Betzel B, Aarts EO, Dogan K, van Laarhoven KJ, Janssen IM, et al. Vitamin and Mineral Deficiencies After Biliopancreatic Diversion and Biliopancreatic Diversion with Duodenal Switch--the Rule Rather than the Exception. Obes Surg. 2015;25(9):1626-32.

17. Brown WA, Ooi G, Higa K, Himpens J, Torres A, on behalf of the IFSOappointed task force reviewing the literature on SADI-S/OADS. Single Anastomosis Duodenal-Ileal Bypass with Sleeve Gastrectomy/One Anastomosis Duodenal Switch (SADI-S/OADS) IFSO Position Statement. Obes Surg. 2018;28:1207-1216.

18. Sánchez-Pernaute A, Rubio Herrera MA, Pérez-Aguirre ME, Talavera P, Cabrerizo L, Matía P, et al. Single Anastomosis Duodeno-lleal Bypass with Sleeve Gastrectomy (SADI-S). One to Three-Year Follow-up. Obes Surg. 2010;20:1720-1726.

19. Moon RC, Kirkpatrick V, Gaskins L, Teixeira AF, Jawad MA. Safety and
Effectiveness of Single- Versus Double-Anastomosis Duodenal Switch at a Single Institution. Surg Obes Relat Dis. 2019:15(2):245-252.

20. Cottam A, Cottam D, Portenier D, Zaveri H, Surve A, Cottam S, et al. A Matched Cohort Analysis of Stomach Intestinal Pylorus Saving (SIPS) Surgery Versus Biliopancreatic Diversion with Duodenal Switch with TwoYear Follow-up. Obes Surg. 2017;27:454-461.

21. Enochs P, Bull J, Surve A, Cottam D, Bovard S, Bruce J, et al. Comparative Analysis of the Single-Anastomosis Duodenal-Ileal Bypass With Sleeve Gastrectomy (SADI-S) to Established Bariatric Procedures: An Assessment of 2-year Postoperative Data Illustrating Weight Loss, Type 2 Diabetes, and Nutritional Status in a Single US Center. Surg Obes Relat Dis. 2020;16(1): 24-33.

22. Cottam A, Cottam D, Roslin M, Cottam S, Medlin W, Richards C, et al. A Matched Cohort Analysis of Sleeve Gastrectomy With and Without $300 \mathrm{~cm}$ Loop Duodenal Switch With 18-Month Follow-Up. Obes Surg. 2016; 26:2363-2369

23. Okafor PN, Lien C, Bairdain S, Simonson DC, Halperin F, Vernon AH, et al. Effect of Vagotomy During Roux-en-Y Gastric Bypass Surgery on Weight Loss Outcomes. Obes Res Clin Pract. 2015;9(3):274-80.

24. Palmisano S, Silvestri M, Giuricin M, Baldini E, Albertario S, Capelli P, et al. Preoperative Predictive Factors of Successful Weight Loss and Glycaemic Control 1 Year After Gastric Bypass for Morbid Obesity. Obes Surg. 2015; 25:2040-2046.

25. Parri A, Benaiges D, Schröder D, Izquierdo-Pulido M, Ramón J, Villatoro M, et al. Preoperative Predictors of Weight Loss at 4 Years Following Bariatric Surgery. Nutr Clin Pract. 2015;30(3):420-4.

26. Contreras JE, Santander C, Court I, Bravo J. Correlation Between Age and Weight Loss After Bariatric Surgery. Obes Surg. 2013;23(8):1286-9.

27. Sanchez Santos R, Corcelles R, Vilallonga Puy R, Delgado Rivilla S, Ferrer $\mathrm{JV}$, Foncillas Corvinos J, et al. Prognostic Factors of Weight Loss After Sleeve Gastrectomy: Multicenter Study in Spain and Portugal. cir esp. 2017; 95(3):135-142.

28. Huang CW, Jiun Tang W, Liu YY, Tsai CY, Yeh TS, Liu KH. Predicted weight loss result of laparoscopic sleeve gastrectomy: Review of the first 82 consecutive patients in an Asian bariatric unit. Asian J Surg. 2019;42:373e378.

29. Cadena-Obando D, Ramírez-Rentería C, Ferreira-Hermosillo A, AlbarránSanchez A, Sosa-Eroza E, Molina-Ayala M, et al. Are there really any predictive factors for a successful weight loss after bariatric surgery? BMC Endocrine Disorders. 2020:20:20.

30. Roslin M, Tugertimur B, Zarabi S, Cottam D. Is There a Better Design for a Bariatric Procedure? The Case for a Single Anastomosis Duodenal Switch. Obes Surg. 2018;28:4077-4086.

31. Vennapusa A, Panchangam BRK, Madivada MSS. A Feasibility Study of Novel "Laparoscopic Sleeve Gastrectomy with Loop Gastroileal Bypass" for Obesity: An Indian Experience. Int Surg. 2017;102(11-12):504-513.

32. Sánchez-Pernaute A, Herrera MA, Pérez-Aguirre ME, Talavera P, Cabrerizo $L$, Matía $P$, et al. Single anastomosis duodeno-ileal bypass with sleeve gastrectomy (SADI-S). One to three-year follow-up. Obes Surg. 2010; 20(12):1720-6 